\title{
Evaluation of the Normal Force of a Planar Actuator
}

\author{
Marilia A. da Silveira, Member, IEEE, Aly F. Flores Filho, and Roberto P. Homrich \\ Federal University of Rio Grande do Sul, Porto Alegre 90.035-190 RS, Brazil
}

\begin{abstract}
This paper describes a method to compute the normal force that acts on the mover of an electromagnetic planar actuator. The method is based on the Maxwell Stress Tensor and depends on the resulting magnetic field. The actuator has a mover with two permanent magnets and a ferromagnetic stator covered by two orthogonal armature coils. The mover is hold by a suspension system with linear bearings that enable bi-directional motion. A normal force is present and it depends on the magnetic flux density distribution produced by the permanent magnets and on the armature magneto motive force, since the latter contributes to that distribution. This paper focuses the analysis of that normal force in order to assess its effects on the actuator and in a way that allows the proper design of the mechanical parts that will be affected by it.
\end{abstract}

Index Terms-Electromagnetic planar actuator, Maxwell Stress Tensor, normal force.

\section{INTRODUCTION}

$\mathbf{S}_{\text {s }}$ OME electromagnetic planar motors rely on a mover with permanent magnets and a stator with orthogonal armature windings mounted around a ferromagnetic armature core. In such devices, the mover can develop planar movement by means of a propulsion force. Furthermore, a normal force is also present. It acts as an attraction force between the permanent magnets and armature ferromagnetic cores. As a result, it loads bearings and shafts that must support the mover and can affect the mobility of the latter. Fig. 1 presents a view of an electromagnetic planar actuator that fits such description. It has a mover with two $\mathrm{Nd}-\mathrm{Fe}-\mathrm{B}$ permanent magnets, each one measuring $25 \times 25 \times 6 \mathrm{~mm}^{3}$, and with $B_{r}=1.24 \mathrm{~T}$, $H_{c} \geq 923.1 \mathrm{kA} / \mathrm{m}$ and $B H_{\max }=295.79 \mathrm{~kJ} / \mathrm{m}^{3}$. A back iron links and holds them. The armature winding comprehends two orthogonal coils, i.e., $x$ and $y$, mounted around the armature core in intercalated layers. Each coil is divided in 12 electrically independent phases or sections. Each phase has 280 turns and the same width as the permanent magnets. The armature core is a slotless iron slab measuring $400 \times 400 \times 15 \mathrm{~mm}^{3}$. The air gap between the armature core and the polar surface of permanent magnets is $14 \mathrm{~mm}$ long. The planar movement occurs when the armature phases located under the permanent magnets are properly fed with currents. A magnetic propulsion force will be created and that pushes the mover accordingly. The normal force depends on the magnetic flux density distribution produced by the permanent magnets and on the armature magneto motive force, since the latter contributes to that distribution. As a result, the effect on the normal force is studied by means of analytical expressions for the magnetic field and experimental results.

\section{Magnetic Field in the Planar Actuator}

The distribution of the magnetic flux density established in the air gap of the planar actuator depends on the magnetic fields produced by the permanent magnets and by the currents in the

Digital Object Identifier 10.1109/TMAG.2005.855153

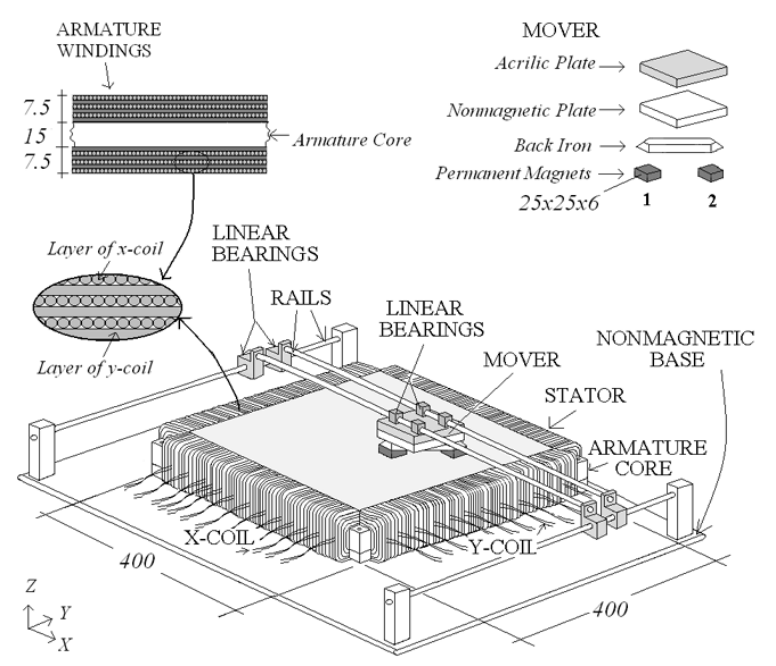

Fig. 1. View of the electromagnetic planar actuator. Units are in millimeter.

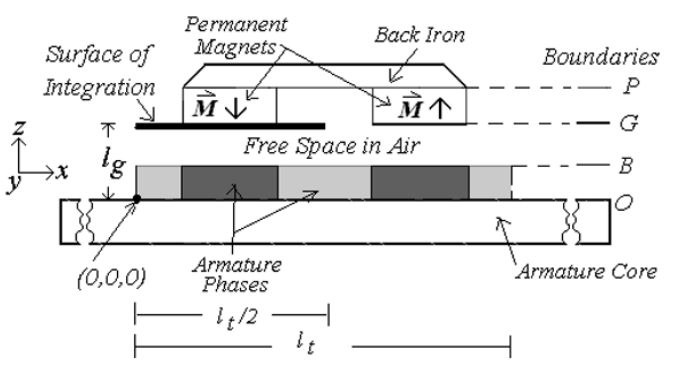

Fig. 2. Frontal view of the planar actuator, its regions, and boundaries. The vertical arrows inside the permanent magnet indicate the magnetization vector direction, $\vec{M}$.

phases of the armature windings. For the purpose of the analysis, the planar actuator was divided into regions and boundaries in order to obtain the equations of the magnetic field and the forces, Fig. 2. The magnetic field due to the permanent magnets was analyzed separately from the magnetic field produced by the armature windings. The soft ferromagnetic material of the planar actuator was considered infinitely permeable. So, it was possible to analyze separately the two magnetic fields, and to carry out the sum of the components of both [1]-[3]. Fig. 3 shows the idealized magnetic flux density distribution produced by the mover and by the currents in the two phases of the $x$-coil 


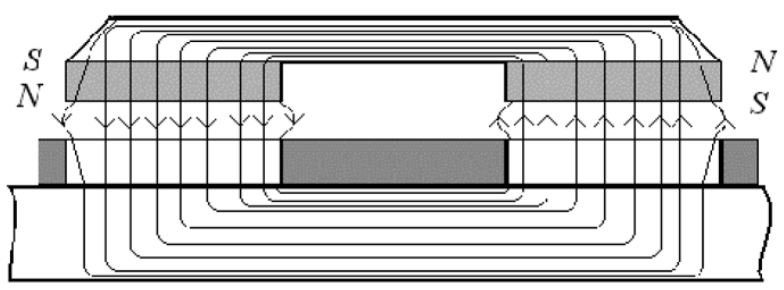

(a)

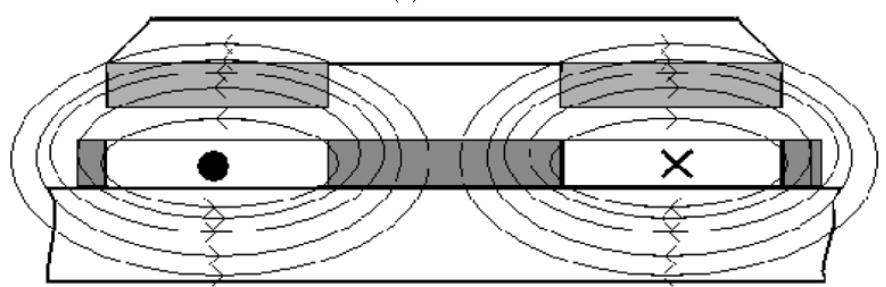

(b)

Fig. 3. Magnetic flux density distribution produced (a) by the permanent magnets and (b) by the currents in the two phases of the $x$-coil located under the permanent magnets.

located under the permanent magnets. The method of analysis considers a tri-dimensional model in rectangular coordinates. The resulting equations of the magnetic field and of the planar propulsion force were presented in previous papers [3], and applicable considerations about the method will be presented here. The effects of eddy currents are not considered, because only the static behavior is studied in this work.

\section{A. Magnetic Field Produced by the Permanent Magnets}

In order to obtain the field equation in the air gap due to the permanent magnets only, an analytical model was developed. Hence, the regions under study are considered free from electric currents, and the magnetic field, $\vec{H}$, can be obtained by using the gradient of the magnetic scalar potential, $\psi$, according to $\vec{H}=$ $-\nabla \psi$. The equation of the magnetic scalar potential in the air gap was obtained by means of the Laplace's equation, $\nabla^{2} \psi=0$. In the permanent magnets region, the magnetic scalar potential must satisfy the Poisson's equation, $\nabla^{2} \psi=\nabla \vec{M}$ [1]-[3]. And by setting a proper integration surface, as show in Fig. 2, the flux density distribution in space is computed and it includes even the fringing flux as the latter in part of the magnetic flux established by the permanent magnets.

\section{B. Magnetic Field Produced by the Armature Windings}

In the static analytical model, only the phases located under the permanent magnets are considered for the production of the magnetic field. The magnetic field produced by the armature windings is obtained by means of the magnetic vector potential $\vec{A}$ related to the current density vector $\vec{J}$. The magnetic flux density vector $\vec{B}$, can be expressed as $\vec{B}=\nabla \times \vec{A}$, where $\vec{A}$ is the magnetic vector potential. In the region where currents are present, $\vec{B}$ can be obtained by means of the Poisson's equation. In the regions free from current, $\vec{B}$ can be obtained by means of the Laplace's equation. The current density vector in the $x$-coil phases is $J_{y} \vec{j}$, whereas in the $y$-coil phases it is $J_{x} \vec{i}$.
Equation (1) results from that analysis. It describes the behavior of the $x$-component of the magnetic flux density vector in the $x$-coil region

$$
\begin{aligned}
& B_{\mathrm{by}_{x}}(x, z)=\sum_{n=1,3, \ldots}^{\infty}\left[\mu_{o} \frac{J_{o} l_{t}^{2}}{(\pi n)^{3}} \cos \left(2 \pi n l_{d} / l_{t}\right)\right. \\
& \left.\times\left(\frac{\left(e^{\lambda\left(2\left(l_{m}+l_{g}\right)-l_{b}\right)}-e^{\lambda l_{b}}\right)}{\left(e^{2 \lambda\left(l_{m}+l_{g}\right)}-1\right)} \lambda \sinh (\lambda z)\right)\right] \sin \left(\frac{2 \pi n}{l_{t}} x\right) .
\end{aligned}
$$

In (1), $B_{\mathrm{by}_{x}}(x, z)$ corresponds to the $x$-component of the magnetic flux density vector in the $x$-coil, generated by $J_{y}, \lambda$ is defined by $\lambda=2 \pi n / l_{t}$, and $J_{o}$ is the peak of the current density vector. Still in (1), $l_{g}$ is the air gap length, $l_{m}$ is the permanent magnet axial length, $l_{d}$ corresponds to the half of the side of the square polar area of each permanent magnet and $l_{t}$ corresponds to the side of the square area considered.

\section{ANAlyticAl EXPRESSION OF the NORMAL ForCE}

For the analysis, the Maxwell Tensor was employed, and it was necessary to know the contribution of the armature phases in the distribution of the magnetic flux density in the air gap. The equation of total force on the mover can be obtained according to $\vec{F}=(1 / \mu) \oint_{S} \vec{T} d \vec{S}$, where $d \vec{S}$ is the surface vector normal to the surface that involves entirely the body where the forces are acting, i.e. the mover of the planar actuator; $\vec{T}$ is the Maxwell Tensor. The $z$-component of the differential of the force is $d F_{z}=(1 / \mu)\left(T_{z x} d y d z+T_{z y} d z d x+T_{z z} d x d y\right)$, where $T_{z x}$ is equal to $B_{z} B_{x}, T_{z y}$ is $B_{z} B_{y}$ and $T_{z z}$ is $B_{z}^{2}-(1 / 2)|B|^{2}$. If an imaginary box involves the mover, the lower surface corresponds to the boundary between the air gap and the lower polar surfaces of the permanent magnets. The top surface is located on $z=+\infty$, where the magnetic field is equal to zero. On the lateral sides of the box, the forces cancel each other. Hence, the normal force that acts on the mover can be obtained by calculating the force that acts on the lower boundary of the imaginary box, showed in Fig. 2, whose differential is $(1 / \mu)\left(T_{z z} d x d y\right)$ [3]. Only the effect of one permanent magnet is taken into account, as long as symmetry is considered. The normal force, $F_{A_{z}}$, can be obtained multiplying by two the result of the integration of the $(1 / \mu)\left(T_{z z} d x d y\right)$ over the surface of integration showed in Fig. 2 according to (2)

$$
F_{A_{z}}=\frac{2}{\mu_{o}} \iint\left(B_{z}^{2}-\frac{1}{2}|B|^{2} d x d y\right)
$$

In (2), the components of the magnetic flux density must be those calculated on the boundary between the air gap and the lower polar surfaces of the permanent magnets, where $z=l_{g}$. That boundary is denoted by $G$. The $x, y$, and $z$ components of the total magnetic flux density vector, $\vec{B}$, result from the sum of the components of the magnetic flux density vectors produced by the permanent magnets and by the currents in the armature phases. Since the ferromagnetic material of the planar actuator was considered infinitely permeable, it was possible to analyze 


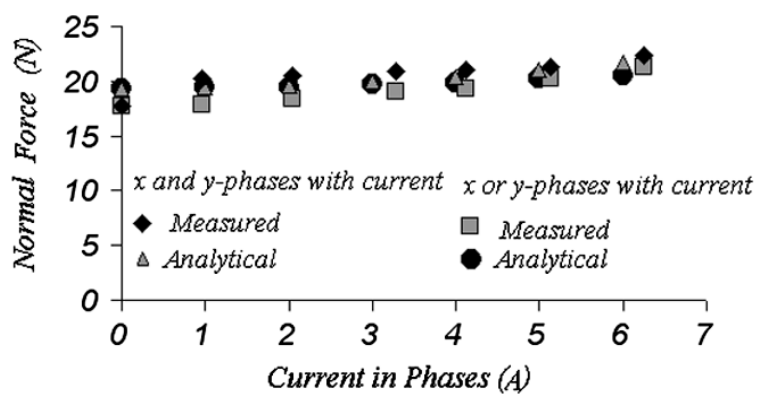

Fig. 4. Normal force versus current in the active phases.

separately the two magnetic fields, and carry out the sum of the components of both, in order to obtain the components of the total magnetic flux density vector. The $x, y$, and $z$-components of the total magnetic flux density vector are $B_{x}^{G}=$ $B_{g_{x}}\left(x, y, l_{g}\right)+B_{g y_{x}}\left(x, l_{g}\right), B_{y}^{G}=B_{g_{y}}\left(x, y, l_{g}\right)+B_{g x_{y}}\left(y, l_{g}\right)$ and $B_{z}^{G}=B_{g_{z}}\left(x, y, l_{g}\right)+B_{g y_{z}}\left(x, l_{g}\right)+B_{g x_{z}}\left(y, l_{g}\right)$, respectively, where $G$ indicates values calculated on the $G$ boundary, $B_{g_{x}}, B_{g_{y}}$ and $B_{g_{z}}$ are the $x, y$, and $z$ components of that magnetic flux density vector, related to the magnetic field produced by the permanent magnets, $B_{g y_{x}}$ and $B_{g y_{z}}$ correspond to the $x$ and $z$ components of the magnetic flux density vector in the region free of currents, generated by $J_{y}$, and $B_{g x_{y}}$ and $B_{g x_{z}}$ correspond to the $y$ and $z$ components of the magnetic flux density vector in the region free of currents, generated by $J_{x}$. Applying those expressions to (2), one can obtain the equation of the normal force that acts over the mover through the $z$-axis, $F_{A_{z}}$. By means of that equation, it is possible to foresee the behavior of the normal force that acts on the mover of the planar actuator. The complete analytical expression of the normal force is presented in (3). Still in (3), $n$, and $m$ are integers, $\gamma$ is defined by $\gamma=\left(2 \pi / l_{t}\right) \sqrt{n^{2}+m^{2}}, M_{o}$ is the remanent magnetization of the permanent magnets, and $\delta=\left(2 \pi m / l_{t}\right)$. Equation (3) comprehends the effects of the permanent magnets and of the currents in the phases of the $\mathrm{x}$ and $\mathrm{y}$ coil of the armature winding located under those permanent magnets.

Fig. 4 presents the graph of analytical normal force when phases of the $\mathrm{x}$ coil and $\mathrm{y}$ coil, located under the permanent magnets, are fed with current, simultaneously or separately. For comparison and validation purposes of the analytical model, measured values are presented, too. They were obtained by means of load cells. For the range of current considered, which is limited by the thermal constraints of the actuator, it affects the normal force in a very limited way

$$
\begin{aligned}
F_{A_{z}}= & \sum_{n, m=1,3, \ldots}^{\infty}\left\{\left[\left(l_{t}^{2} M_{o} \frac{\left(e^{\gamma l_{g}}-e^{\gamma\left(2 l_{m}+l_{g}\right)}\right)}{\left(e^{2 \gamma\left(l_{m}+l_{g}\right)}-1\right)}\right.\right.\right. \\
& \left.\times \cos \left(2 \pi n l_{d} / l_{t}\right) \cos \left(2 \pi m l_{d} / l_{t}\right)\right)^{2} \\
& \times\left(-\left(\frac{1}{2 \pi^{2} n \cdot m}\left(e^{\gamma l_{g}}+e^{-\gamma l_{g}}\right)\right)^{2}\right. \\
& \left.\left.+\left(\left(\frac{1}{\pi l_{t} \gamma}\left(e^{\gamma l_{g}}-e^{-\gamma l_{g}}\right)\right)^{2}\left(\frac{1}{n^{2}}+\frac{1}{m^{2}}\right)\right)\right)\right] \\
& +\left[( \frac { J _ { o } l _ { t } ^ { 2 } } { 4 \pi ^ { 2 } } ) ^ { 2 } \left[\left[\left(\frac{\cos \left(2 \pi n l_{d} / l_{t}\right)}{n^{2}} \cdot \frac{\left(e^{\lambda l_{b}}-e^{-\lambda l_{b}}\right)}{\left(e^{\lambda 2 l_{t}}-1\right)}\right)^{2}\right.\right.\right. \\
& \left.\times\left(-\left(e^{\lambda l_{g}}+e^{\lambda\left(2 l_{t}-l_{g}\right)}\right)^{2}+\left(e^{\lambda l_{g}}-e^{\lambda\left(2 l_{t}-l_{g}\right)}\right)^{2}\right)\right] \\
& +\left[\left(\frac{\cos \left(2 \pi m l_{d} / l_{t}\right)}{m^{2}} \cdot \frac{\left(e^{\delta l_{b}}-e^{-\delta l_{b}}\right)}{\left(e^{\delta 2 l_{t}}-1\right)}\right)^{2}\right. \\
& \times\left(-\left(e^{\delta l_{g}}+e^{\delta\left(2 l_{t}-l_{g}\right)}\right)^{2}\right. \\
& \left.\left.\left.\left.+\left(e^{\delta l_{g}}-e^{\delta\left(2 l_{t}-l_{g}\right)}\right)^{2}\right)\right]\right]\right\} .
\end{aligned}
$$

\section{CONCLUSION}

The analytical expressions of the magnetic field in the air gap of the planar actuator allowed one to compute the behavior of the normal force over the mover. And that is important to the development of the suspension system that holds the mover and must not jeopardise the planar movement of the latter. The analytical and the experimental results of the normal force showed a good agreement, which validates the former ones computed.

\section{REFERENCES}

[1] J. R. Melcher, Continuum Electromechanics. Cambridge, MA: MIT Press, 1981.

[2] D. L. Trumper, M. E. Williams, and T. H. Nguyen, "Magnet arrays for synchronous machines," in Proc. IEEE IAS 28th Annual Meeting, 1993, pp. 216-223.

[3] A. F. F. Filho, A. A. Susin, and M. A. da Silveira, "An analytical method to predict the static performance of a planar actuator," IEEE Trans. Magn., vol. 39, no. 9, pp. 3364-3366, Sep. 2003.

Manuscript received January 21, 2005. 